\title{
A footnote to Nelson's interpretation of the two-slit experiment
}

\author{
dedicated to Francesco Guerra on the occasion of his 60th birthday \\ Michele Pavon \\ Dipartimento di Matematica Pura e Applicata \\ Università di Padova \\ via Belzoni 7, and ISIB-CNR \\ 35131 Padova, Italy \\ pavon@math.unipd.it
}

August 1, 2018

\begin{abstract}
We seek to complement Nelson's work on the two-slit experiment by showing that the two-slit process, whose density exhibits the characteristic interference pattern, may be obtained as the model after the beam has reached the screen by means of a variational mechanism. The one-slit process, modeling the beam before it reaches the screen, plays the role of a reference model.
\end{abstract}

Keywords: two-slit experiment, stochastic mechanics

PACS number: $03.65 . \mathrm{Bz}$

\section{Introduction}

When a beam of electrons originating from a source $\mathcal{S}$ goes through a crystal, one observes a diffraction pattern resembling that characteristic of interference in wave motion. In a celebrated gedanken experiment $[1, \mathrm{pp} .2-5]$, the 
crystal is replaced by a screen with two slits. As it is well-known, according to quantum mechanics, this is an instance of a superposition of two states. The two possibilities of going through the top or the bottom slit interfere. Hence, the probability of arrival at the second screen is not simply the sum of the probabilities of coming through the top and the bottom slit.

In $[2,3]$, Edward Nelson made a remarkable calculation showing that, according to his version of stochastic mechanics, the observed interference effect can be explained within the frame of classical probability. In particular, the probability of arrival is indeed the sum of the probabilities of coming through the top and the bottom slit.

In this note we seek to complement what Nelson did. We model the emission from the source through a suitable Gaussian process (one-slit process). We then take the latter as a reference process in a variational problem that takes into account the presence of the screen with the two slits. The solution of the variational problem, suitably extending on the results of $[4,5]$, is the stochastic process that Nelson has analyzed (two-slit process) whose density profile is familiar in wave interference.

\section{Elements of the Nelson-Guerra stochastic mechanics}

Nelson's stochastic mechanics is a quantization procedure for classical dynamical systems based on diffusion processes. Following some early work by Feynes [6] and others, Nelson and Guerra elaborated a clean formulation starting from 1966 [7, 8, 9] and references therein, showing that the Schrödinger equation could be derived from a continuity type equation plus a Newton type law, provided one accepted a certain definition for the stochastic acceleration. The Newton-Nelson law was later shown to follow, in analogy to classical mechanics, from a Hamilton-like stochastic variational principle $[10,11]$. Other versions of the variational principle have been proposed in $[2,12,13,14]$.

Consider the case of a nonrelativistic particle of mass $m$. Let $\left\{\psi(x, t) ; t_{0} \leq\right.$ $\left.t \leq t_{1}\right\}$, satisfying the Schrödinger equation

$$
\frac{\partial \psi}{\partial t}=\frac{i \hbar}{2 m} \Delta \psi-\frac{i}{\hbar} V(x) \psi,
$$


be such that

$$
\|\nabla \psi\|_{2}^{2} \in L_{\text {loc }}^{1}\left[t_{0},+\infty\right) .
$$

This is Carlen's finite action condition. Under these hypotheses, the Nelson measure $P$ may be constructed on path space, [15],[16], [12, Chapter IV], and references therein. Namely, letting $\Omega:=\mathcal{C}\left(\left[t_{0}, t_{1}\right], \mathbb{R}^{n}\right)$ the $n$-dimensional continuous functions on $\left[t_{0}, t_{1}\right]$, under the probability measure $P$, the canonical coordinate process $x(t, \omega)=\omega(t)$ is an $n$-dimensional, Markov, finite-energy diffusion process $\left\{x(t) ; t_{0} \leq t \leq t_{1}\right\}$, called Nelson's process, having (forward) Ito differential

$$
d x(t)=\left[\frac{\hbar}{m} \nabla(\Re \log \psi(x(t), t)+\Im \log \psi(x(t), t))\right] d t+\sqrt{\frac{\hbar}{m}} d w(t),
$$

where $w$ is a standard, $n$-dimensional Wiener process. Moreover, the probability density $\rho(\cdot, t)$ of $x(t)$ satisfies Born's relation

$$
\rho(x, t)=|\psi(x, t)|^{2}, \quad \forall t \in\left[t_{0}, t_{1}\right] .
$$

\section{Nelson's treatment of the two-slit experi- ment}

We recall some essential points in Nelson's analysis in $[2,3]$. Let $x \in \mathbb{R}^{3}$, and consider free motions, i.e. $V \equiv 0$, in a frame of reference comoving with the beam. For the sake of simplicity, take $m=1, \hbar=1$ and $t_{0}=0$. The slits are located at $\pm a$, where $a$ is a vector in $\mathbb{R}^{3}$. Let

$$
\psi_{0}(x)=\left(\frac{\lambda}{\pi}\right)^{1 / 4} \exp \left(-\frac{|x|^{2}}{2 \lambda}\right)
$$

be the initial condition at time $t_{0}$. Then, the corresponding solution of

$$
\frac{\partial \psi}{\partial t}=\frac{i \hbar}{2 m} \Delta \psi
$$

is

$$
\psi_{0}(x, t)=\left(\frac{\lambda}{\pi}\right)^{1 / 4}(\lambda+i t)^{-1 / 2} \exp \left(-\frac{|x|^{2}}{2(\lambda+i t)}\right)
$$


Nelson calls the one-slit process the Gaussian process associated to this evolution whose forward and backward drifts are given by

$$
b_{+}(x, t)=\frac{t-\lambda}{\lambda^{2}+t^{2}} x, \quad b_{-}(x, t)=\frac{t+\lambda}{\lambda^{2}+t^{2}} x .
$$

He then takes as initial condition for (1) the vector

$$
\psi_{1}(x)=\gamma\left(\psi_{0}(x-a)+\psi_{0}(x+a)\right),
$$

where $\gamma$ is a constant (close to $1 / \sqrt{2}$ if $|a|$ is large with respect to $\lambda$ ) to make $\psi_{1}$ a unit vector in $L^{2}$. By the linearity of the Schrödinger equation, the corresponding solution is

$$
\psi_{1}(x, t)=\gamma\left(\psi_{0}(x-a, t)+\psi_{0}(x+a, t)\right) .
$$

Nelson goes ahead and computes the forward drift of the two-slit process corresponding to $\psi_{1}$. As only the direction joining the slits is of interest, the problem can be reduced to a one-dimensional problem (hence, from now on, $a, x \in \mathbb{R})$. He finds that, for small times, the drift is nearly the same as for the one-slit process. For larger times, however, the drift of the twoslit process becomes enormous in certain regions, repelling particles from there. As a result, the probability density of the two-slit process after some time exhibits alternate peaks and valleys resembling those observed in wave interference.

\section{A stochastic variational principle}

Let $\bar{v}$ be the velocity of the beam, let $d$ be the distance between the source and the screen with the two slits. Let $T=d / \bar{v}$ be the time it takes the beam to reach the screen. In order to keep $t=0$ the time when the beam is at the screen, we assume that the beam is emitted from the source $\mathcal{S}$ at time $-T$. The one-slit process with $\lambda$ very small associated to $\psi_{0}(x, T+t)$ as in (6) models effectively the beam of particles between times $-T$ and 0 . Suppose now we impose at time $t=0$ the probability density

$$
\rho_{0}(x)=\frac{1}{2}\left(\left|\psi_{0}(x-a)\right|^{2}+\left|\psi_{0}(x+a)\right|^{2}\right) .
$$

We define $\mathcal{X}_{0}$ to be the family of real-valued stochastic processes $\{x(t) ;-T \leq$ $t \leq 0\}$ with continuous paths and satisfying the following properties: 
1. $x(t)$ has a nowhere vanishing probability density $\rho(x, t)$ of class $C^{1}$ for all $t \in[-T, 0]$ with marginal probability density $\rho_{0}$ at time 0 ;

2. $E\left\{\int_{-T}^{0}\left|\frac{\partial}{\partial x} \log \rho(x(t), t)\right|^{2} d t\right\}<\infty$;

3. there exists for each $t \in[-T, 0]$ a random variable $v(t)$, called the current drift, such that, if $f(x, t)$ is of class $C^{2,1}$ satisfying

$$
E\left\{\int_{-T}^{0}\left|\frac{\partial}{\partial x} f(x(t), t)\right|^{2} d t\right\}<\infty
$$

we have

$$
\frac{d E\{f(x(t), t)\}}{d t}=E\left\{\left(\frac{\partial}{\partial t}+v(t) \frac{\partial}{\partial x}\right) f(x(t), t)\right\}
$$

Notice that property (iii) is satisfied by a rather large class of processes including differentiable processes, finite-energy processes with constant diffusion coefficient $[17,2]$. It also includes Markovian diffusion processes with local diffusion coefficient given the results in $[21,20,19,18]$. The current drift $v$ is just the semi-sum of the forward and backward drifts. Let $\mathcal{V}$ denote the family of finite-energy stochastic processes on $[-T, 0]$. In the following variational problem, we take one-slit process $\left\{x_{r}(t+T) ;-T \leq t \leq 0\right\}$ with small $\lambda$ associated to $\left\{\psi_{0}(x, T+t) ;-T \leq t \leq 0\right\}$ as reference model on the time interval $[-T, 0]$. Write $\psi_{0}(x, T+t)=\rho_{r}(x, t) \exp \left[\frac{i}{\hbar} S_{r}(x, t)\right]$. Rather than setting up a variational problem with complex-valued drifts of the form $v-i u$ as in Section VIII in [4] and [5], we formulate here a problem with distinct real-valued velocities $v$ and $u$ as in Section IV in [4] and [22]. For $\left(x, v, u^{\prime}\right) \in\left(\mathcal{X}_{0}, \mathcal{V}, \mathcal{V}\right)$, define the functional

$$
\begin{aligned}
& I(x, v, u):=E\left\{\int _ { - T } ^ { 0 } \left[\frac{1}{2} m\left(v(t)-\frac{1}{m} \frac{\partial}{\partial x} S_{r}(x(t), t)\right)^{2}\right.\right. \\
& \left.\left.-\frac{\hbar^{2}}{8 m}\left(u^{\prime}(t)-\frac{\partial}{\partial x} \log \rho_{r}(x(t), t)\right)^{2}\right] d t-S_{r}(x(0), 0)\right\} .
\end{aligned}
$$

Notice that the integrand is the same as in the Guerra-Morato action [11] where differences of drifts replace drifts. We consider the stochastic differential game 


$$
\min _{x \in \mathcal{X}_{0}} \max _{v \in \mathcal{V}} \min _{u^{\prime} \in \mathcal{V}} I\left(x, v, u^{\prime}\right)
$$

subject to

$$
\begin{aligned}
& v(\cdot) \text { is the current drift of } x, \\
& u^{\prime}(t)=\frac{\partial}{\partial x} \log \rho(x(t), t) \forall t,
\end{aligned}
$$

where $\rho(\cdot, t)$ is the probability density of $x(t)$. We say that $\left(x^{*}, v^{*}, u^{*}\right)$ is a saddle-point equilibrium solution of the game if for all $\left(x_{1}, v^{*}, u^{\prime}\right)$ and $\left(x_{2}, v, u^{\prime *}\right)$ in $\mathcal{X}_{0} \times \mathcal{V} \times \mathcal{V}$ satisfying the constraints we have

$$
I\left(x_{1}, v^{*}, u^{\prime}\right) \leq I\left(x^{*}, v^{*}, u^{\prime *}\right) \leq I\left(x_{2}, v, u^{\prime *}\right) .
$$

Let $F:\left(\mathbb{R} \times\left[t_{0}, t_{1}\right]\right) \rightarrow \mathbb{R}$ be of class $C^{1}$ satisfying the condition in property iii). Let $\lambda:\left(\mathbb{R} \times\left[t_{0}, t_{1}\right]\right) \rightarrow \mathbb{R}$ be of class $C^{1}$. For such a pair $(F, \lambda)$, define

$$
\begin{aligned}
& \Lambda^{F, \lambda}\left(x, v, u^{\prime}\right)=E\{F(x(0), 0)-F(x(-T),-T) \\
& \left.-\int_{-T}^{0}\left\{\left[\frac{\partial F}{\partial t}+v \frac{\partial}{\partial x} F\right](x(t), t)-\lambda(x(t), t)\left[u^{\prime}(t)-\frac{\partial}{\partial x} \log \rho(x(t), t)\right]\right\} d t\right\} .
\end{aligned}
$$

Observe that for all triples $\left(x, v, u^{\prime}\right)$ in $\mathcal{X}_{0} \times \mathcal{V} \times \mathcal{V}$ satisfying the constraints, we have $\Lambda^{F, \lambda}\left(x, v, u^{\prime}\right)=0$. Obviously, if $\left(x, v, u^{\prime}\right) \in\left(\mathcal{X}_{0} \times \mathcal{V} \times \mathcal{V}\right)$ satisfying the constraints is a saddle-point solution for $\left(I+\Lambda^{F, \lambda}\right)$, then it also solves the original problem with cost function $I$.

It is possible to rewrite $\Lambda^{F, \lambda}$ in a form more suited for our purposes using argument similar to that in [11]. Observe that, for any $x \in \mathcal{X}_{0}$, we have

$$
\begin{aligned}
& E\left\{\lambda(x(t), t) \frac{\partial}{\partial x} \log \rho(x(t), t)\right\}=\int_{\mathbb{R}^{n}} \lambda(x, t) \frac{\partial}{\partial x} \rho(x, t) d x \\
& =-\int_{\mathbb{R}^{n}} \frac{\partial}{\partial x} \lambda(x, t) \rho(x, t) d x=-E\left\{\frac{\partial}{\partial x} \lambda(x(t), t)\right\}
\end{aligned}
$$

where, in the integration by parts, we have used the natural boundary condition at infinity for $\rho(\cdot, t)$. Thus, our $\Lambda^{F, \lambda}$ functionals now have the form

$$
\begin{aligned}
& \Lambda^{F, \lambda}\left(x, v, u^{\prime}\right)=E\{F(x(0), 0)-F(x(-T),-T) \\
& \left.-\int_{-T}^{0}\left\{\left[\frac{\partial F}{\partial t}+v(t) \frac{\partial}{\partial x} F\right](x(t), t)-\lambda(x(t), t) \cdot u^{\prime}(t)-\frac{\partial}{\partial x} \lambda(x(t), t)\right\} d t\right\} .
\end{aligned}
$$


The variational analysis now follows the same lines as in [22]. Consider now the unconstrained problem

$$
\min _{x \in \mathcal{X}_{0}} \min _{v \in \mathcal{V}} \max _{u^{\prime} \in \mathcal{V}}\left(I+\Lambda^{F, \lambda}\right)\left(x, v, u^{\prime}\right) .
$$

For each fixed $x \in \mathcal{X}_{0}$, and each $t \in[-T, 0]$, we study the finite-dimensional problem

$$
\begin{aligned}
\min _{v \in \mathbb{R}} \max _{u^{\prime} \in \mathbb{R}} & \left\{\frac{1}{2} m\left(v-\frac{1}{m} \frac{\partial}{\partial x} S_{r}(x(t), t)\right)^{2}-\frac{\hbar^{2}}{8 m}\left(u^{\prime}-\frac{\partial}{\partial x} \log \rho_{r}(x(t), t)\right)^{2}\right. \\
- & \left.\frac{\partial F}{\partial t}(x(t), t)-v \frac{\partial}{\partial x} F(x(t), t)+\lambda(x(t), t) u^{\prime}+\frac{\partial}{\partial x} \lambda(x(t), t)\right\} .
\end{aligned}
$$

We get the optimality conditions

$$
\begin{gathered}
\left.v_{x}^{*}(t)=\frac{1}{m} \frac{\partial}{\partial x} S_{r}(x(t), t)\right)+\frac{1}{m} \frac{\partial}{\partial x} F(x(t), t), \\
\left.u_{x}^{\prime *}(t)=\frac{\partial}{\partial x} \log \rho_{r}(x(t), t)\right)+\frac{4 m}{\hbar^{2}} \lambda(x(t), t) .
\end{gathered}
$$

Remark 4.1 If a stochastic process with the prescribed $v_{x}^{*}(t)$ and $u_{x}^{*}(t)$ does exist, then the first optimality condition implies that it is a Markov process and the second that $\lambda$ is given by

$$
\lambda(x, t)=\frac{\hbar^{2}}{4 m} \frac{\partial}{\partial x} \log \left(\frac{\rho_{x}^{*}}{\rho_{r}}\right)(x, t),
$$

where $\rho_{x}^{*}(x, t)$ denotes the probability density of $x^{*}(t)$.

Notice that $v^{x}$ and $u^{\prime x}$ belong to $\mathcal{V}$. Consider next the minimization of

$$
\begin{array}{r}
\left(I+\Lambda^{F, \lambda}\right)\left(x, v_{x}^{*}, u_{x}^{\prime *}\right)=E\{F(x(0), 0)-F(x(-T),-T) \\
+\int_{-T}^{0}\left[-\frac{\partial F}{\partial t}(x(t), t)-\frac{1}{2 m}\left(\frac{\partial}{\partial x} F(x(t), t)\right)^{2}-\frac{1}{m} \frac{\partial}{\partial x} S_{r}(x(t), t)\right) \frac{\partial}{\partial x} F(x(t), t) \\
\left.\left.\left.\frac{2 m}{\hbar^{2}} \lambda(x(t), t)^{2}+\frac{\partial}{\partial x} \log \rho_{r}(x(t), t)\right) \cdot \lambda(x(t), t)+\frac{\partial}{\partial x} \cdot \lambda(x(t), t)\right] d t\right\}
\end{array}
$$


on the space $\mathcal{X}_{0}$. We wish to choose $F$ and $\lambda$ such that the functional becomes constant with respect to the process $x \in \mathcal{X}_{0}$. Suppose that the pair $(F, \lambda)$ satisfies on $\mathbb{R} \times[-T, 0]$ the equation

$$
\frac{\partial F}{\partial t}+\frac{1}{2 m}\left(\frac{\partial}{\partial x} F\right)^{2}+\frac{1}{m} \frac{\partial}{\partial x} S_{r} \cdot \frac{\partial}{\partial x} F-\frac{2 m}{\hbar^{2}} \lambda^{2}-\frac{\partial}{\partial x} \log \rho_{r} \cdot \lambda-\frac{\partial}{\partial x} \cdot \lambda=0,
$$

and the boundary condition $F\left(x, t_{0}\right)=-S_{r}(x, 0)$. With this choice of $(F, \lambda)$, $\left.\left(I+\Lambda^{F, \lambda}\right)\left(x, v_{x}^{*}, u_{x}^{*}\right)\right) \equiv E\{F(x(0), 0)\}$ which is constant on $\mathcal{X}_{0}$ (all processes have the same marginal density at $t=0)$. Hence, any $x$ in $\mathcal{X}_{0}$ solves with $\left(v^{x}, u^{\prime x}\right)$, the unconstrained problem.

\section{Solution to the variational problem}

In view of Remark 4.1, we now write

$$
\lambda(x, t)=\frac{\hbar^{2}}{2 m} \frac{\partial}{\partial x} G(x, t)
$$

for some scalar $C^{1}$ function $G$. Equation (9) then becomes

$$
\frac{\partial F}{\partial t}+\frac{1}{2 m}\left(\frac{\partial}{\partial x} F\right)^{2}+\frac{1}{m} \frac{\partial}{\partial x} S_{r} \cdot \frac{\partial}{\partial x} F-\frac{\hbar^{2}}{2 m}\left[\left(\frac{\partial}{\partial x} G\right)^{2}+\frac{\partial^{2}}{\partial x^{2}} G+\frac{\partial}{\partial x} \log \rho_{r} \frac{\partial}{\partial x} G\right]=0 .
$$

In the ideal case where $\frac{\partial}{\partial x} S_{r} \equiv 0$ and $\rho_{r}=$ const, this equation reduces to one of the Madelung equations. If $F$ and $G$ may be found satisfying (10) with $F\left(x, t_{0}\right)=-S_{r}(x, 0)$, then any $x$ in $\mathcal{X}_{\rho_{1}}$ solves the unconstrained problem. If we can find one $x^{*}$ in $\mathcal{X}_{\rho_{1}}$ that also satisfies the constraints

$$
\begin{array}{r}
\left.v_{x}^{*}(t)=\frac{1}{m} \frac{\partial}{\partial x} S_{r}(x(t), t)\right)+\frac{1}{m} \frac{\partial}{\partial x} F(x(t), t) \text { is the current drift of } x \\
\frac{\partial}{\partial x} \log \rho^{*}(x(t), t)=\frac{\partial}{\partial x} \log \rho_{r}(x(t), t)+2 \frac{\partial}{\partial x} G(x, t) \forall t,
\end{array}
$$

then it solves the original stochastic differential game. In that case, we define

$$
S^{*}(x, t):=S_{r}(x, t)+F(x, t),
$$


so that $\left.v_{x}^{*}(t)=\frac{1}{m} \frac{\partial}{\partial x} S^{*}(x(t), t)\right)$ and we have

$$
G(x, t)=\frac{1}{2} \log \frac{\rho_{x}^{*}}{\rho_{r}}(x, t) .
$$

Suppose that we have found such a process $x^{*}$ with density $\rho^{*}$. As observed before, it is a Markov process and so is the Nelson reference process $x_{r}$. Then the corresponding Fokker-Planck (continuity) equations read

$$
\begin{aligned}
& \frac{\partial \rho^{*}}{\partial t}+\frac{\partial}{\partial x}\left(\frac{1}{m} \frac{\partial}{\partial x} S^{*} \rho^{*}\right)=0 \\
& \frac{\partial \rho_{r}}{\partial t}+\frac{\partial}{\partial x}\left(\frac{1}{m} \frac{\partial}{\partial x} S_{r} \rho_{r}\right)=0 .
\end{aligned}
$$

Using these equations, and relations (11)-(12), we get that $F$ and $G$ must satisfy another equation

$$
\begin{gathered}
\frac{\partial G}{\partial t}=\frac{\partial\left(\frac{1}{2} \log \frac{\rho_{x}^{*}}{\rho_{r}}\right)}{\partial t}=\frac{1}{2}\left[-\frac{1}{\rho *} \frac{\partial}{\partial x}\left(\frac{1}{m} \frac{\partial}{\partial x} S^{*} \rho^{*}\right)+\frac{1}{\rho_{r}} \frac{\partial}{\partial x}\left(\frac{1}{m} \frac{\partial}{\partial x} S_{r} \rho_{r}\right)\right] \\
=-\frac{1}{2 m} \frac{\partial^{2}}{\partial x^{2}}\left(S^{*}-S_{r}\right)-\frac{1}{2 m} \frac{\partial}{\partial x} S^{*} \frac{\partial}{\partial x} \log \frac{\rho_{x}^{*}}{\rho_{r}}-\frac{1}{2 m} \frac{\partial}{\partial x}\left(S^{*}-S_{r}\right) \frac{\partial}{\partial x} \log \rho_{r} \\
=-\frac{1}{2 m} \frac{\partial^{2}}{\partial x^{2}} F-\frac{1}{m} \frac{\partial}{\partial x} F \frac{\partial}{\partial x} G+\frac{1}{m} \frac{\partial}{\partial x} S_{r} \frac{\partial}{\partial x} G-\frac{1}{2 m} \frac{\partial}{\partial x} F \frac{\partial}{\partial x} \log \rho_{r} .
\end{gathered}
$$

Thus, $F$ and $G$ also satisfy

$$
\frac{\partial G}{\partial t}+\frac{1}{m} \frac{\partial}{\partial x} F \frac{\partial}{\partial x} G+\frac{1}{2 m} \frac{\partial^{2}}{\partial x^{2}} F+\frac{1}{2 m} \frac{\partial}{\partial x} F \cdot \frac{\partial}{\partial x} \log \rho_{r}-\frac{1}{m} \frac{\partial}{\partial x} S_{r} \frac{\partial}{\partial x} G=0 .
$$

In the ideal case where $\frac{\partial}{\partial x} S_{r} \equiv 0$ and $\rho_{r}=$ const, this equation reduces to the other Madelung equation. Moreover,

$$
G(x, 0)=\frac{1}{2} \log \frac{\rho_{0}}{\rho_{r}}(x, 0) .
$$

Define $\theta=\exp \left(G+\frac{i}{\hbar} F\right)$. Then, equations (10)-(15) imply that $\theta$ satisfies

$$
\frac{\partial \theta}{\partial t}+\left(\frac{1}{m} \frac{\partial}{\partial x} S_{r}+\frac{\hbar}{i m} \frac{\partial}{\partial x} R_{r}\right) \frac{\partial}{\partial x} \theta-\frac{i \hbar}{2 m} \frac{\partial^{2}}{\partial x^{2}} \theta=0
$$

Define now $\psi_{n}:=\left(\rho^{*}\right)^{1 / 2} \exp \frac{i}{\hbar} S^{*}=\psi_{r} \theta$. It follows from (5) and (17) that $\psi_{n}$ also satisfies $(5)$ and $\psi_{n}(x, 0)=\left(\rho_{0}(x)\right)^{1 / 2}$. 


\section{Conclusion}

Hence, we find that the solution to the stochastic differential game is the Nelson process associated to another solution of the same Schrödinger equation (5), with the probability density $\rho_{0}$ at time 0 and $S^{*}(x, 0)=0$ (so that $v(0)=0)$. In other words, the variational principle shows that the process with density $\rho_{0}$ at time 0 and $v(0)=0$ that replaces the one-slit reference process is precisely the two-slit process studied by Nelson in $[2,3]$.

As observed by Nelson in [3], the probability density $\rho_{0}(x)$ can, for all practical purposes, be replaced by $\left|\psi_{1}(x, 0)\right|^{2}$, where $\psi_{1}$ was defined in (8). Thus, the new process, obtained from the variational principle, will exhibit, after a time sufficiently large with respect to $a$, the typical interference pattern in the probability density.

We have made here no attempt at a physical interpretation of the variational mechanism. We refer the reader to [23, 24, 25], and references therein,

for a discussion of various crucial topics in stochastic mechanics from the physical standpoint.

\section{Acknowledgment}

I wish to thank Laura Morato for several useful conversations on the two-slit experiment.

\section{References}

[1] R. Feynman and A.Hibbs, Quantum Mechanics and Path integrals (McGraw-Hill, New York, 1965).

[2] E. Nelson, Quantum Fluctuations, (Princeton University Press, Princeton, 1985).

[3] E. Nelson, Stochastic mechanics and random fields, in Ècole d'Ètè de Probabilitès de Saint-Flour XV-XVII, Lecture Notes in Mathematics, ed. P. L. Hennequin (Springer-Verlag, New York, 1988), Vol.1362, pp. 428450.

[4] M. Pavon, J. Math. Physics 40, 5565 (1999). 
[5] M. Pavon, Quantum Schrödinger bridges, in Directions in Mathematical Systems Theory and Optimization, ed. A. Rantzer et al., (Springer-Verlag, Berlin, 2002), pp. 227-238.

[6] I. Fenyes, Z. Physik 132, 81 (1952).

[7] E. Nelson, Phys. Rev. 150, 1079 (1966).

[8] E. Nelson, Dynamical Theories of Brownian Motion. (Princeton University Press, Princeton, 1967).

[9] F. Guerra, Phys.Rep. 77, 263 (1981).

[10] K. Yasue, J. Functional Analysis 41327 (1981).

[11] F. Guerra and L. Morato, Phys.Rev.D 27, 1774 (1983).

[12] Ph. Blanchard, Ph. Combe and W. Zheng. Math. and Physical Aspects of Stochastic Mechanics. Lect. Notes in Physics vol. 281, (Springer-Verlag, New York, 1987).

[13] M. Pavon, J. Math. Phys. 36, 6774 (1995).

[14] H. H. Rosenbrock, IEEE Trans. Aut. Contr. 54, 73 (2000).

[15] E. Carlen, Comm. Math. Phys., 94, 293 (1984).

[16] R. Carmona, in Taniguchi Symp. PMMP Katata (1985), pp. 55-81.

[17] H. Föllmer, in Stochastic Processes - Mathematics and Physics, Lecture Notes in Mathematics (Springer-Verlag, New York,1986), Vol. 1158, pp. $119-129$.

[18] U.G.Haussmann and E.Pardoux, The Annals of Probability 14, 1188 (1986).

[19] L. M. Morato, J. Math. Phys. 23, 1020 (1982).

[20] M.Nagasawa, Kodai Math.Sem.Rep. 13, 235 (1961).

[21] E. Nelson, Duke Math. J. 25, 671 (1958).

[22] M. Pavon, in Mathematical Theory of Networks and Systems, Proc. MTNS 1998 Conf., ed. A. Beghi et al., (Il Poligrafo, Padova, Italy, 1998), pp. 851-854.

[23] F. Guerra, in The Foundation of Quantum Mechanics (Kluwer, Amsterdam, 1995).

[24] F. Guerra, in New perspectives in the physics of mesoscopic systems, ed. S. De Martino et al. (World Scientific, Singapore, 1997), 133.

[25] N. Cufaro Petroni and L. Morato, J. Phys. A 335833 (2000). 